\title{
The Use of Ostrich Eggs for In Ovo Research: Making Preclinical Imaging Research Affordable and Available
}

\author{
Martin Freesmeyer ${ }^{1}$, Christian Kuehnel ${ }^{1}$, Thomas Opfermann ${ }^{1}$, Tobias Niksch ${ }^{1}$, Steffen Wiegand ${ }^{1}$, Ronny Stolz ${ }^{2}$, \\ Ralph Huonker ${ }^{3}$, Otto W. Witte ${ }^{3,4}$, and Thomas Winkens ${ }^{1}$ \\ ${ }^{I}$ Clinic of Nuclear Medicine, Jena University Hospital, Jena, Germany; ${ }^{2}$ Leibniz Institute of Photonic Technology, Jena, Germany; \\ ${ }^{3}$ Biomagnetic Center, Hans Berger Department of Neurology, Jena University Hospital, Jena, Germany; and ${ }^{4}$ Hans Berger \\ Department of Neurology, Jena University Hospital, Jena, Germany
}

In ovo studies are a valuable option in preclinical research, but imaging studies are severely limited by the costs of dedicated equipment needed for small-sized eggs. We sought to verify the feasibility of using larger, ostrich, eggs (Struthio camelus) for imaging on the PET/CT scanners used for routine clinical investigations. Methods: Ostrich eggs were incubated until shortly before hatching, prepared for intravitelline venous injection of contrast medium or radiotracer, and imaged using native $\mathrm{CT}$, contrast-enhanced $\mathrm{CT}$, and PET/CT. Any technical adaptations that were needed to improve the outcome were noted. Results: Of the 34 eggs initially incubated, 12 became fully available for imaging of embryonal development. In ovo imaging with conventional PET/CT not only was feasible but also provided images of good quality, including on dynamic PET imaging. Conclusion: In ovo imaging with ostrich eggs and routine clinical scanners may allow broader application of this field of preclinical research, obviating costly dedicated equipment and reducing the number of animals needed for classic animal research. Further experiments are warranted to refine this novel approach, especially to reduce motion artifacts and improve monitoring of viability.

Key Words: in ovo imaging; functional topography; embryonal development; ostrich

J Nucl Med 2018; 59:1901-1906

DOI: 10.2967/jnumed.118.210310

$\mathbf{I}$ ovo imaging of chicken embryos is an established preclinical method for research in oncology, toxicology, and pharmacology $(1-3)$. Imaging of tumor cell formations and vascularization has been evaluated using chorion-allantois membrane models of chicken eggs as reported previously (4-6). In addition, chicken eggs have been investigated using MRI to visualize developmental processes in ovo, predominantly considering brain development (7-9).

Because of the small size of chicken eggs, however, this method requires expensive, dedicated imaging equipment (i.e., small-animal CT, MRI, PET, or SPECT), as well as the availability of adequate premises and well-trained personnel (10). These prerequisites substantially

Received Feb. 21, 2018; revision accepted May 31, 2018.

For correspondence or reprints contact: Martin Freesmeyer, Clinic of Nuclear Medicine, Jena University Hospital, Am Klinikum 1, 07743 Jena, Germany.

E-mail: martin.freesmeyer@med.uni-jena.de

Published online Jun. 22, 2018.

COPYRIGHT (C) 2018 by the Society of Nuclear Medicine and Molecular Imaging. limit broad application of in ovo research, which has the benefit of reducing the number of animal tests and meeting the requirements of modern animal research (11).

We explored the possibility of performing in ovo imaging without dedicated techniques by using ostrich (Struthio camelus) eggs (i.e., eggs that are considerably larger than conventional chicken eggs) along with conventional PET/CT, a technique routinely used for clinical diagnostics $(10,12-14)$. The goal was to determine whether in ovo PET/CT imaging of ostrich eggs is feasible in terms of viability monitoring, fenestration, anesthesia, vessel catheterization, and image acquisition and reconstruction.

\section{MATERIALS AND METHODS}

\section{Ethics}

This embryo study did not qualify as an animal research study according to the Federal German Animal Protection Act. Registration took place with the Office for Consumer Protection of the Thuringia State, registration number 22-2684-04-02-114/16.

\section{Egg Sample}

The size of the egg is the first requisite for possible use in routine imaging. Ostrich eggs appeared to be good candidates for this purpose, given their average size of $20 \times 15 \mathrm{~cm}$ (Fig. 1A) and the possibility of obtaining a sufficiently precise depiction of the embryonal anatomic structures within the resolution limits of common imaging methods ( $1 \mathrm{~mm}$ or less for MRI and CT, a few $\mathrm{mm}$ for PET, $\mathrm{mm}$ to $\mathrm{cm}$ for SPECT). Ostrich eggs are commonly available at German farms from March to September. For this study, the eggs were obtained either as freshly fertilized (development stage, 0 ; cost, €20 [\$23]) or as already incubated (development stage, days 1-36; cost, €50-€130 [\$58$\$ 151]$ ). They were then placed in an incubator (Grumbach Compact MP GTFS84; ProCon Automatic Systems GmbH \& Co. KG) at $36.4^{\circ} \mathrm{C}-$ $36.5^{\circ} \mathrm{C}$ and $30 \%$ humidity, and the position of the egg was changed once or twice a day during the incubation period (15-17). These conditions allowed continuous development of the embryo, given that artificial incubation may have a failure rate of $20 \%-50 \%$ (18). A regular check of viability by candling was performed to avoid spread of infection from dead embryos. This simple visual monitoring method was used because no other reliable methods are described or commercially available.

\section{Egg Preparation}

To allow for intravascular administration of contrast medium and radiotracer into ostrich egg embryos, it was first necessary to identify suitable vessels in the amniotic membrane (Figs. 1B-1D). This was performed by means of a light-intensive candling lamp (Tempo no. 119 [Brecker Ltd. \& Co. KG] or Powerlux Eggtester 4.5 VDC [Lyon 


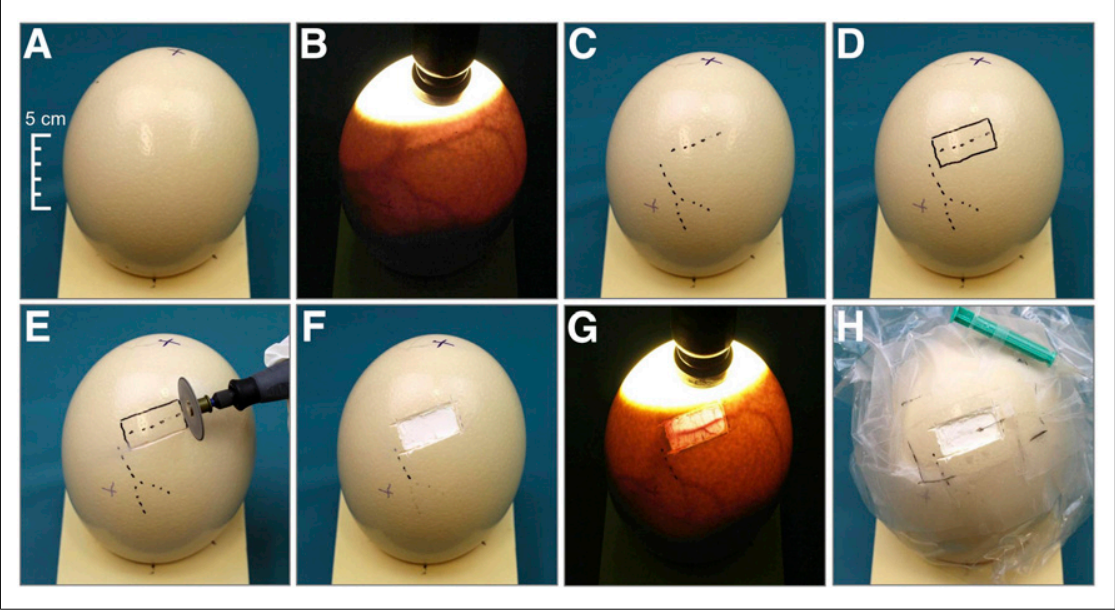

FIGURE 1. Preparation of ostrich egg (day 36 of incubation). (A) Vertically placed ostrich egg stabilized on polyurethane foam base. (B) Light-intensified candling lamp enhancing air cell, below which egg content and vessels become visible. (C) Vessel profiles drawn on eggshell. (D) Suitable place for fenestration marked. (E) Fenestration by means of rotating cutter. (F) Visibility of chorionallantois membrane after removal of eggshell. (G) Repeated candling of vessels. (H) Puncture and insertion of 27-gauge cannula; $3.5 \%$ mixture of isoflurane and air in plastic bag sealed around egg immobilizes embryo, and operating window is kept open for full interventional access.

Technologies Inc.]), which allows a diaphanoscopylike depiction through the eggshell (Fig. 1B). After a suitably large vessel had been located, a rectangular piece of the 2-mm-thick eggshell $(2.5 \times 5 \mathrm{~cm})$ was removed with a rotating cutter (Dremel 3000; Bosch Powertools B.V.) (Fig. 1E), taking care to maintain the integrity of the inner shell membrane, that is,

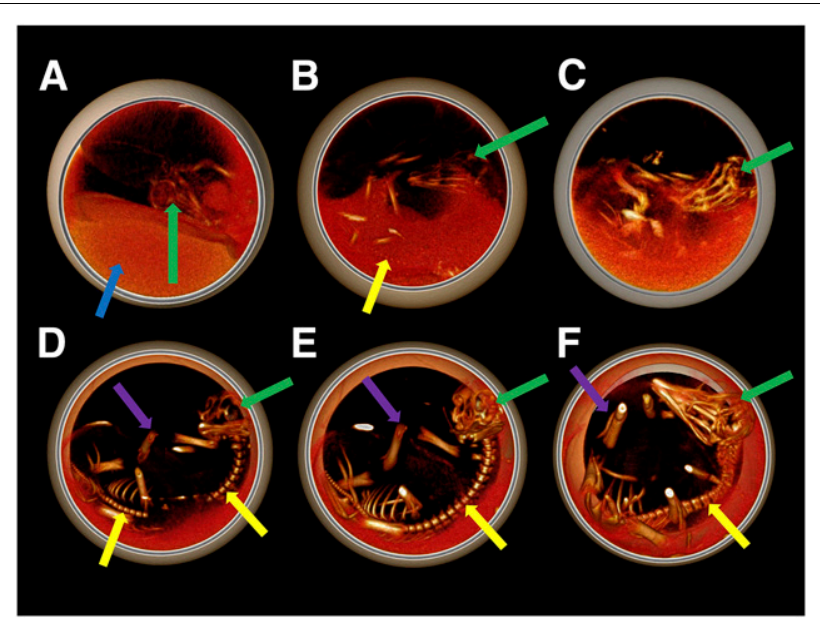

FIGURE 2. Serial 3-dimensional volume-rendered CT transaxial images of developing ostrich embryo, with visualization of bone tissue. Size is measured from vertex to end of tail. (A) On day 24 of incubation, size is $121 \mathrm{~mm}$ and skull and yolk are seen. (B) On day 29, size is $149 \mathrm{~mm}$ and faint osseous structures with vertebrae are seen. (C) On day 32 , size is $186 \mathrm{~mm}$ and larger bone structures can be seen than in $B$; bone calcification increases with increasing incubation time. (D) On day 34 , size is $207 \mathrm{~mm}$ and embryo occupies more than half of egg. Vertebrae and head are clearly visible, and lower extremities can also be identified. (E) On day 36, size is $228 \mathrm{~mm}$ and progressive embryonal growth is seen. (F) On day 38 , size is $241 \mathrm{~mm}$ and a decrease in surrounding space is seen, along with further embryonal growth. Green arrows = head; blue arrow = yolk; yellow arrows = vertebrae; purple arrows $=$ lower extremities. the chorion-allantois membrane. Thereafter, a vitelline vein was punctured with a thin 27-gauge cannula, again with the help of a candling lamp. The cannula was fixed to the eggshell with tape (Figs. 1F-1H). This access allowed injection of CT contrast medium or radiotracer by means of a plastic tube (800/100/100; Smiths Medical International Ltd.). Formation of clots was prevented by flushing with heparin.

\section{Anesthesia}

Anesthesia is particularly relevant for nuclear medicine investigations, since motion is associated with imaging artifacts. Immobilization of the embryo is thus a prerequisite for good-quality images (13). When chicken eggs are applied for in ovo research, anesthetics in gas form are commonly used (13). Isoflurane (Piramal Critical Care Deutschland $\mathrm{GmbH}$ ) at a maximum of $4 \%$ was thus chosen for this study and was delivered using a standard vaporizer (Vaporizer Medimorph System; VEB MLW Medizintechnik). To ensure a high concentration and constant diffusion of the anesthetic gas into the embryonal blood, the egg was placed in a plastic bag for the whole duration of the contrast injection and image acquisition (Fig. 1H).

\section{Investigations}

Imaging was performed with a clinical PET/CT scanner (Biograph mCT40; Siemens). In addition to obtaining native CT scans, enhancement with contrast medium and radiotracers was also used (Fig. 2). The number of investigated eggs, the activities used, and other relevant parameters are shown in Table 1. Further experiments were performed using different tracers (e.g., ${ }^{18} \mathrm{~F}$-fluoroethyl-tyrosine, ${ }^{68} \mathrm{Ga}$-tetraazacyclododecane-tetraacetic-octreotide, and ${ }^{68} \mathrm{Ga}$-prostate-specific membrane antigen); however, these tracers were not investigated systematically in this study. Some eggs were investigated sequentially, either with longlived radiotracers ( 1 injection, several scans) or with short-lived radiotracers (several injections, several scans; Table 2).

\section{Imaging Parameters}

The eggs were kept vertical using a polyurethane base (Necuron 100; Necumer GmbH) (Fig. 1). First, a full-dose CT scan was acquired for the purpose of attenuation correction $(120 \mathrm{kV}, 200 \mathrm{mAs}, 0.6-\mathrm{mm}$ slice thickness, 0.3-mm increment, reconstruction kernel $\mathrm{H}_{2} \mathrm{O}$ ). The $\mathrm{CT}$ data were reconstructed via filtered backprojection. Finally, a PET acquisition was obtained with 1 bed position $(21.8-\mathrm{cm}$ transaxial field of view). The reconstruction was performed with a gauss filter ( $2.0 \mathrm{~mm}$ in full width at half maximum) in an iterative manner (4 orders, 12 subsets) with a zoom of 2 and a matrix of $512 \times 512$. A dynamic PET scan in list mode was also recorded, as shown in Supplemental Videos 1 and 2 (supplemental materials are available at http://jnm.snmjournals.org). The first of these videos is a dynamic list-mode PET/CT acquisition of an ${ }^{18} \mathrm{~F}-\mathrm{NaF}$ study (40 MBq) on day 38 of incubation. The data acquisition was started $4 \mathrm{~min}$ after injection and ended $64 \mathrm{~min}$ after injection. The time after the start of acquisition is shown at the bottom. Thirty-one time frames of variable duration (10-300 s) were reconstructed. The embryo was anesthetized $82 \mathrm{~min}$ before injection using $3.5 \%$ isoflurane. On early images (13 min after the start of the acquisition), $\mathrm{NaF}$ was clearly visible within the vitelline vessels (orange arrow) and within the embryonal circulation (i.e., heart, red arrow). On late images (3-60 min after the start 
TABLE 1

Imaging Tests Performed on the 34 Eggs

\begin{tabular}{|c|c|c|}
\hline Test & $n$ & $\begin{array}{l}\text { Injected volume } \\
\text { or activity }\end{array}$ \\
\hline $\begin{array}{l}\text { CT scans, } \\
\text { native }\end{array}$ & 204 & - \\
\hline $\begin{array}{l}\text { CT scans, } \\
\text { contrast- } \\
\text { enhanced }\end{array}$ & 2 & $\begin{array}{l}\text { Volume: mean } \pm \mathrm{SD} \\
2.5 \pm 0.7 \mathrm{~mL} \text {; range, } \\
2.0-3.0 \mathrm{~mL}\end{array}$ \\
\hline PET scans & 18 & $\begin{array}{l}\text { Volume: mean } \pm \mathrm{SD} \\
1.3 \pm 0.8 \mathrm{~mL} \text {; range, } \\
\text { 0.6-3.0 mL; median, } 1.0 \mathrm{~mL}\end{array}$ \\
\hline $\begin{array}{l}{ }^{18} \mathrm{~F}-\mathrm{FDG} \\
\mathrm{PET} / \mathrm{CT} \\
\text { scans }\end{array}$ & 8 & $\begin{array}{l}\text { Activity: mean } \pm \mathrm{SD} \\
32.8 \pm 4.4 \mathrm{MBq} \\
\text { range, } 29.1-40.1 \mathrm{MBq} ; \\
\text { median, } 30.3 \mathrm{MBq}\end{array}$ \\
\hline $\begin{array}{r}{ }^{18} \mathrm{~F}-\mathrm{NaF} \\
\text { scans }\end{array}$ & 1 & Activity: mean, $38.0 \mathrm{MBq}$ \\
\hline $\begin{array}{r}{ }^{124} \text { I-Nal } \\
\text { scans }\end{array}$ & $6^{*}$ & $\begin{array}{l}\text { Activity: mean } \pm \mathrm{SD} \\
0.8 \pm 0.3 \mathrm{MBq} \\
\text { range, } 0.54-0.99 \mathrm{MBq}\end{array}$ \\
\hline
\end{tabular}

${ }^{* 124}$ I-Nal was injected once into 2 eggs; afterward, 4 datasets were acquired for first egg and 2 datasets for second egg at different time points, considering relatively long half-life of $100 \mathrm{~h}$. of the acquisition start), tracer is seen to gradually increase in the bone tissue. The embryo skull (green arrow), vertebrae (yellow arrow), and lower extremities (purple arrow) show intense uptake at the end of the acquisition. Slight movement $(\sim 6 \mathrm{~mm})$ of the embryonal extremities can be seen 50-55 min after the start of the acquisition.

The second video is a dynamic list-mode PET/CT acquisition of an ${ }^{18}$ F-FDG study $(29 \mathrm{MBq})$ on day 32 of incubation. Data acquisition began immediately after tracer injection and ended $60 \mathrm{~min}$ after injection. Thirty-one time frames of variable duration (10-300 s) were reconstructed. The embryo was anesthetized $94 \mathrm{~min}$ before injection using 3.5\% isoflurane. Intense tracer accumulation was seen within the vitelline vessels (1-3 min after injection), and tracer distribution was rapid, with depiction of the embryonal body and head (arrow). Immobilization was insufficient despite a maximal dose of isoflurane. The head could clearly be seen to move in multiple directions at 25$60 \mathrm{~min}$ (maximum movement of head and neck, 30 and $9 \mathrm{~mm}$, respectively). The embryo motion limits the application of a volume of interest for a semiquantitative determination of tissue uptake using SUVs, because the underlying CT-based attenuation correction is erroneous if the structures move between the CT and PET acquisitions.

\section{Disposal of Eggs}

Ostrich eggs usually hatch between days 39 and 42 of incubation; therefore, the embryos were sacrificed by freezing on day 38 at the latest (19).

\section{RESULTS}

In total, 34 eggs were investigated, 15 of which did not contain an embryo (fertilization or incubation failure), as shown by CT scans between days 6 and 12. These eggs were thus excluded from

TABLE 2

Sequential Quantitative PET Data

\begin{tabular}{|c|c|c|c|c|c|c|c|c|c|c|}
\hline \multirow[b]{3}{*}{ Tracer } & \multirow{3}{*}{$\begin{array}{c}\text { Day of } \\
\text { incubation }\end{array}$} & \multirow{3}{*}{$\begin{array}{c}\text { Injected } \\
\text { activity } \\
(\mathrm{MBq})\end{array}$} & \multirow{3}{*}{$\begin{array}{c}\text { Time } \\
\text { between } \\
\text { injection and } \\
\text { acquisition }\end{array}$} & \multirow{3}{*}{$\begin{array}{c}\text { Scan } \\
\text { duration }\end{array}$} & \multicolumn{6}{|c|}{ Organ activity $(\mathrm{kBq} / \mathrm{mL})$} \\
\hline & & & & & \multicolumn{2}{|c|}{ Liver/thyroid* } & \multicolumn{2}{|c|}{ Brain } & \multicolumn{2}{|c|}{ Yolk } \\
\hline & & & & & Max & Mean & Max & Mean & Max & Mean \\
\hline \multirow[t]{4}{*}{${ }^{18} \mathrm{~F}-\mathrm{FDG}$} & 27 & 30.3 & $28 \mathrm{~min}$ & $10 \mathrm{~min}$ & 160.4 & 133.4 & 151.0 & 136.3 & 0 & 0 \\
\hline & 28 & 36.7 & $29 \mathrm{~min}$ & $10 \min$ & 163.8 & 139.4 & 155.3 & 140.3 & 0.2 & 0.1 \\
\hline & 29 & 40.1 & $30 \mathrm{~min}$ & $10 \min$ & 178.8 & 159.1 & 159.8 & 136.2 & 0 & 0 \\
\hline & 32 & 34.8 & $30 \mathrm{~min}$ & $10 \mathrm{~min}$ & $5,225.3$ & 729.6 & 0 & 0 & 0 & 0 \\
\hline \multirow[t]{4}{*}{${ }^{124}$ I-Nal } & 29 & 0.99 & $2 \mathrm{~h}$ & $10 \mathrm{~min}$ & 4.3 & 2.0 & 1.1 & 0.5 & 0.01 & 0 \\
\hline & 30 & 0.99 & $20 \mathrm{~h}$ & $10 \mathrm{~min}$ & 3.0 & 1.4 & 0.6 & 0.2 & 0.6 & 0.1 \\
\hline & 31 & 0.99 & $44 \mathrm{~h}$ & $10 \min$ & 7.9 & 2.4 & 0.4 & 0.2 & 0.9 & 0.2 \\
\hline & 33 & 0.99 & $96 \mathrm{~h}$ & $10 \mathrm{~min}$ & 10.9 & 6.2 & 0.2 & 0.1 & 1.6 & 0.4 \\
\hline
\end{tabular}

${ }^{*}$ Data are liver for ${ }^{18} \mathrm{~F}-\mathrm{FDG}$ and thyroid for ${ }^{124} \mathrm{I}-\mathrm{Nal}$.

Mean data are for $1.5-\mathrm{mL}$ spheric volume of interest for ${ }^{18} \mathrm{~F}-\mathrm{FDG}$ in liver, $1.0-\mathrm{mL}$ spheric volume of interest for ${ }^{18} \mathrm{~F}-\mathrm{FDG}$ and ${ }^{124} \mathrm{I}-\mathrm{Nal}$ in brain, $5.0-\mathrm{mL}$ spheric volume of interest for ${ }^{18} \mathrm{~F}-\mathrm{FDG}$ and ${ }^{124} \mathrm{I}-\mathrm{Nal}$ in yolk, and isocontour volume of interest (threshold, $35 \%$ of maximum activity) for ${ }^{124} \mathrm{I}-\mathrm{Nal}$ in thyroid.

Sequential ${ }^{18} \mathrm{~F}-\mathrm{FDG}$ PET/CT with 4 injections of ${ }^{18} \mathrm{~F}-\mathrm{FDG}$ on different days produces homogeneous results for representative volumes of interest in first 3 studies. Fourth study, without brain uptake, is associated with embryo's death between days 29 and 32 of incubation. High liver uptake is attributable to adjacent blood-pool activity in heart and thus represents artifact. Sequential ${ }^{124}$ I-Nal PET/CT with 1 injection of $\mathrm{Nal}$ and 4 scans on different days produces increasing thyroid activity over time, attributable to continuous iodine trapping within thyroid. Gradually decreasing brain activity is presumably due to reduction of blood-pool activity over time. Increasing yolk activity over time is most likely attributable to unspecific tracer distribution and partial excretion within this compartment. 


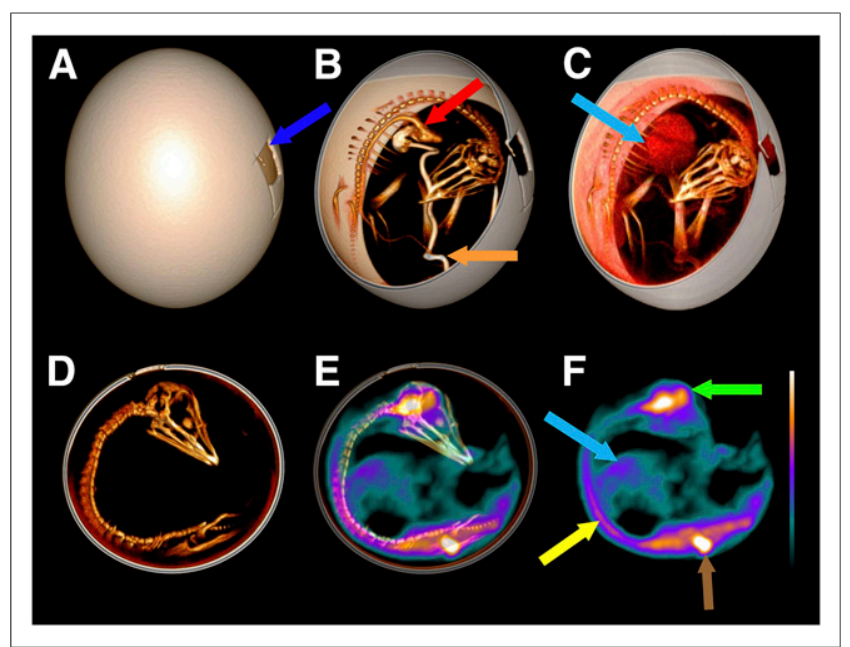

FIGURE 3. Three-dimensional volume-rendered CT and PET/CT images on day 32 of ostrich egg incubation. (A) Access window (arrow). (B) Dual-clipping plane revealing inner structures, with texture mapping to show bone tissue. This early sequence image was obtained after administration of $1 \mathrm{~mL}$ of standard contrast agent (Ultravist 300; Bayer) into vitelline vein. As vitelline circulation empties into omphalomesenteric vein, these vessels (orange arrow) and both atria are clearly visible. Heart ventricles and aorta are also faintly enhanced (red arrow). (C) Image acquired later than but under same conditions as for B, faintly showing liver (arrow) and blood pool. (D-F) CT (D), ${ }^{18} \mathrm{~F}-\mathrm{FDG}$ PET/CT $(\mathrm{E})$, and ${ }^{18} \mathrm{~F}-\mathrm{FDG}$ PET $(\mathrm{F})$ images with slim dual-clipping plane and texture mapping, showing eggshell and axial skeleton of embryo, with intense homogeneous uptake within brain (green arrow), allantois (brown arrow), and bone marrow (yellow arrow), and low-intensity uptake in liver (blue arrow).

the analyses. The remaining 19 eggs underwent multiple CT scanning to verify continuous development (Fig. 2).

Two of 19 eggs presented a secretion of thick fluid associated with arrest of development. The remaining 17 eggs were investigated at a mean development of $34.6 \pm 2.5 \mathrm{~d}$ (range, 31-38 d; median, $35 \mathrm{~d}$ ).

The chorion-allantois membrane of 3 of 17 eggs was damaged during fenestration; thus, no vessel catheterization was possible. The catheterization failed in 2 of the remaining 14 eggs because of formation of a hematoma that limited the visibility of the vitelline vein. As a result, 12 eggs were fully available for imaging investigations.

The prepared access was used for multiple injections in 5 eggs, so that injection of short-lived radiotracers to investigate the development stage could be performed with the same or a different tracer. Table 1 shows the radiotracer parameters for ${ }^{18} \mathrm{~F}-\mathrm{NaF},{ }^{18} \mathrm{~F}-$ FDG, and ${ }^{124} \mathrm{I}-\mathrm{NaI}$. Exemplary quantitative PET data are shown in Table 2. Estimated effective doses ranged from 2.4 to $6.4 \mathrm{mGy}$ for CT imaging.

The potential of 3-dimensional imaging that combines the highresolution anatomy of CT with the functional topography of PET is detailed in Figure 3. Dynamic list-mode imaging with ${ }^{18} \mathrm{~F}-\mathrm{NaF}$ and ${ }^{18} \mathrm{~F}-\mathrm{FDG}$ is shown in Supplemental Videos 1 and 2, respectively.

Concerning the issue of viability monitoring, several methods were tested (acoustic detection of cardiac tones via auscultation, cardiotocogram, electrocardiogram, or pressure measurements) but did not prove suitable. Only cardiac pulsation could be identified, via a modified egg-electrocardiogram (Buddy MK2; Avitronics) based on infrared phase delay caused by the blood flow through the embryo's vessels. However, the repositioning of the egg until a good signal was obtained was lengthy and error- prone, and an electrocardiogram could be derived for only 3 of 10 eggs. Another promising approach, although not fully implemented, was the use of a magnetogram. This biomagnetic method is based on the detection of magnetic field changes arising from changes in cardiac activity or embryo movements. This method has the advantage of detecting in parallel the viability and movement of the embryos.

Ostrich embryos showed regular motion and change of position starting from day 20 of development, as could be seen with CT series and dynamic PET investigations. Indeed, despite the use of maximum concentrations of isoflurane $(3.5 \%-4 \%)$, the immobilization of the embryos during PET scanning remained insufficient in 10 of 12 cases (Supplemental Videos 1 and 2).

\section{DISCUSSION}

The present study showed that in ovo imaging with conventional PET/CT not only is a feasible option but also is associated with good image quality (Figs. 2 and 3). The use of readily available imaging modalities for in ovo research with ostrich embryos offers several advantages, as shown in Table 3. Also, given the clarity of images observed with conventional PET/CT, it is conceivable that the use of ostrich eggs is transferable to other common modalities of clinical imaging, for example, SPECT, CT, MRI, and ultrasonography. As shown in Supplemental Videos 1 and 2, dynamic radionuclide studies using ${ }^{18} \mathrm{~F}-\mathrm{NaF}$ and ${ }^{18} \mathrm{~F}-\mathrm{FDG}$ are also feasible, augmenting the area of application.

An additional major benefit of the increased availability of in ovo research with ostrich eggs is the possibility of reducing the number of experiments needed on fully developed animals (11) and limiting the use of mammals. Indeed, chicken in ovo imaging has already been proposed as a feasible alternative to imaging studies on mice (10). Even genome modification strategies have been described for avian species, conceivably allowing for in ovo knock-out disease models, as these are widely used in mice and rats (20). Furthermore, in ovo research enables studies evaluating processes that occur during embryonal development, which are difficult to perform on mammals. Figure 2 shows sufficient visualization of embryonal structures starting from the second half of development. Imaging studies performed at earlier stages of development are presumably limited by small organ size (18).

If in ovo research with ostrich eggs is used for imaging, the matter of reproducible quantification needs to be considered. Preliminary PET data analysis on sequential ${ }^{18} \mathrm{~F}-\mathrm{FDG}$ and ${ }^{124} \mathrm{I}-$ NaI studies produced homogeneous results (Table 2), indicating that this concept might be suitable for quantification as well. However, this aspect has not been investigated systematically yet, and further studies addressing this issue are planned.

At an activity concentration of $13.5 \mathrm{kBq} / \mathrm{mL}$, the recovery coefficients for ${ }^{18} \mathrm{~F}$ ranged from 0.15 to 0.84 for small-volume spheres of 3.95-15.43 $\mathrm{mm}$ in diameter, respectively. Regarding image quality, conventional scanners are outperformed by dedicated small-animal devices. The PET/CT scanner used in the present study is characterized by a resolution of $4.2 \times 4.2 \times 4.5 \mathrm{~mm}$, and the nanoScan PET/CT (PET 8/2; Mediso) offers a resolution of $0.8 \times 0.8 \times 0.85 \mathrm{~mm}(21)$. Considering that the organs of ostrich embryos are naturally larger than those of chicken embryos (e.g., liver size: 15 vs. $\left.2 \mathrm{~cm}^{3}\right)(18,22)$, it is conceivable that imaging is comparable. This aspect has to be evaluated in future studies. Obviously, because of the small gantry size of $98.6 \mathrm{~mm}$, the 
TABLE 3

Advantages and Disadvantages of In Ovo Imaging with Ostrich Eggs

\begin{tabular}{|c|c|}
\hline Advantage & Disadvantage \\
\hline $\begin{array}{l}\text { High availability (no dedicated small-animal imaging } \\
\text { device necessary) }\end{array}$ & $\begin{array}{l}\text { Higher cost per ostrich egg (€20-130 [\$23-\$151]) } \\
\text { than per chicken eggs }\end{array}$ \\
\hline $\begin{array}{l}\text { Low costs for equipment, premises, and personnel } \\
\text { (if clinical scanner used for humans is available on site) }\end{array}$ & $\begin{array}{l}\text { Shorter availability of ostrich eggs (March-September) } \\
\text { than of chicken eggs }\end{array}$ \\
\hline No need for mouse or rat husbandry & $\begin{array}{l}\text { Lower spatial resolution than for dedicated } \\
\text { small-animal devices }\end{array}$ \\
\hline $\begin{array}{l}\text { Not considered animal testing, simplifying study } \\
\text { design planning }\end{array}$ & $\begin{array}{l}\text { Restricted experimentation time (afternoon or } \\
\text { evening, after all patients have been examined) }\end{array}$ \\
\hline \multicolumn{2}{|l|}{ Larger organ size than for chicken embryos } \\
\hline \multicolumn{2}{|l|}{ Lower radiation exposure than for small-animal CT devices } \\
\hline $\begin{array}{l}\text { Shorter acquisition and higher temporal resolution than } \\
\text { for flat-panel small-animal CT devices }\end{array}$ & \\
\hline
\end{tabular}

small-animal device does not allow the investigation of ostrich eggs (21).

The use of ostrich eggs required several technical adaptions of the methods described for chicken eggs. Although the issues of eggshell access, vessel catheterization, and reconstruction parameters were addressed in this study, several issues need further investigation. The monitoring of embryo viability by means of conventional commercial methods (Buddy MK2), for example, was not satisfactory. A potential improvement in this case is the use of a magnetometer, which would allow the monitoring of cardiac activity in a noninvasive manner (23). Another issue is the anesthesia, because most of the embryos moved despite maximum anesthesia levels using isoflurane. The maximum isoflurane concentration was limited by the specific vaporizer used. We conclude that isoflurane is not suitable for effectively reducing motion, conceivably because of its limited diffusion through the eggshell. Since movement is a major drawback for imaging experiments, this problem requires a systematic search for a general solution that guarantees artifact-free images. The use of intravenous anesthetics and muscle relaxants might represent a suitable approach to this problem. The negative influence of motion on image quality has also been described for chicken eggs investigated with smallanimal MRI scanners, which are prone to motion artifacts in a similar way unless ultrafast gradients are used $(8,24)$. Both MRI and PET/CT imaging protocols rely on data acquisition over several minutes, emphasizing the need for effective immobilization. From a technical point of view, list-mode data acquisition allows for motion correction in PET/CT datasets; however, erroneous attenuation correction caused by incongruence between CT and PET and, thus, imprecise SUV measurements, needs to be considered. Effective doses by CT are comparably low, considering published doses ranging from $5 \mathrm{mGy}$ to $4.5 \mathrm{~Gy}$ for small-animal CT devices (25). Therefore, radiation attributable to $\mathrm{CT}$ in this concept $(6.4 \mathrm{mGy})$ presumably does not influence the embryo's viability.

Regarding limitations, the present study was a proof-of-concept investigation of the feasibility of an imaging model in ovo and was not designed to cover the arising issues in a systematic way. Also, the imaging investigations focused exclusively on the use of ostrich embryos, and the results are thus not transferable to mammals, that is, humans, without restrictions. Furthermore, it is not yet clear that an embryo is a suitable model for imaging processes that occur in adults. Further disadvantages of this model are described in Table 3.

\section{CONCLUSION}

The proposed method of in ovo imaging of ostrich embryos by means of routine scanners allows a broader application of this field of preclinical research, since the techniques available to date require the use of dedicated and costly imaging scanners. This novel method may also help reduce the number of tests based on classic animal research. Further experiments are warranted to refine the in ovo approach with ostrich eggs, particularly regarding improvement of viability monitoring, motion reduction, and artifact reduction.

\section{DISCLOSURE}

No potential conflict of interest relevant to this article was reported.

\section{ACKNOWLEDGMENTS}

Dr. Ernesta Palombo-Kinne is gratefully acknowledged for language assistance with the manuscript. Prof. Dr. Hans-Joachim Mentzel, Prof. Dr. Jürgen R. Reichenbach, Dr. Daniel Güllmar, and Dr. Karl-Heinz Herrmann (Institute of Diagnostic and Interventional Radiology, Jena University Hospital) are gratefully acknowledged for technical advice on MRI. We thank Dr. Sabine Bischoff (Institute of Lab Animal Sciences and Welfare, Jena University Hospital) and Prof. Dr. Christian Hübner (Institute of Human Genetics, Jena University Hospital) for general advice on animal experiments. Furthermore, we thank Prof. Ekkehard Schleußner and Prof. Dr. Uwe Schneider (Department of Obstetrics, Jena University Hospital) for advice on monitoring embryonal viability.

\section{REFERENCES}

1. Czarnecki CM, Sujarit VK. Effects of furazolidone in the early chick embryo. Poult Sci. 1979;58:988-990. 
2. Deryugina EI, Quigley JP. Chick embryo chorioallantoic membrane model systems to study and visualize human tumor cell metastasis. Histochem Cell Biol. 2008;130:1119-1130.

3. Rashidi H, Sottile V. The Chick embryo: hatching a model for contemporary biomedical research. Bioessays. 2009;31:459-465.

4. Woloszyk A, Liccardo D, Mitsiadis TA. Three-dimensional imaging of the developing vasculature within stem cell-seeded scaffolds cultured in ovo. Front Physiol. 2016;7:146.

5. Kivrak Pfiffner F, Waschkies C, Tian Y, et al. A new in vivo MRI method to noninvasively monitor and quantify the perfusion capacity of 3D-biomaterials grown on the chorioallantoic membrane of chick embryos. Tissue Eng Part C Methods. 2014;21:339-346.

6. Oppitz M, Pintaske J, Kehlbach R, Schick F, Schriek G, Busch C. Magnetic resonance imaging of iron-oxide labeled SK-Mel 28 human melanoma cells in the chick embryo using a clinical whole body MRI scanner. MAGMA. 2007;20: $1-9$.

7. Peebles DM, Dixon JC, Thornton JS, et al. Magnetic resonance proton spectroscopy and diffusion weighted imaging of chick embryo brain in ovo. Brain Res Dev Brain Res. 2003;141:101-107.

8. Bain MM, Fagan AJ, Mullin JM, McNaught I, McLean J, Condon B. Noninvasive monitoring of chick development in ovo using a 7T MRI system from day 12 of incubation through to hatching. J Magn Reson Imaging. 2007;26:198-201.

9. Boss A, Oppitz M, Wehrl HF, et al. Measurement of T1, T2, and magnetization transfer properties during embryonic development at 7 Tesla using the chicken model. J Magn Reson Imaging. 2008;28:1510-1514.

10. Haller S, Ametamey SM, Schibli R, Muller C. Investigation of the chick embryo as a potential alternative to the mouse for evaluation of radiopharmaceuticals. Nucl Med Biol. 2015;42:226-233.

11. Kilkenny C, Browne WJ, Cuthill IC, Emerson M, Altman DG. Improving bioscience research reporting: the ARRIVE guidelines for reporting animal research. PLoS Biol. 2010;8:e1000412.

12. Gebhardt P, Wurbach L, Heidrich A, et al. Dynamic behaviour of selected PET tracers in embryonated chicken eggs. Rev Esp Med Nucl Imagen Mol. 2013;32:371-377.

13. Heidrich A, Wurbach L, Opfermann T, Saluz HP. Motion-artifact-free in vivo imaging utilizing narcotized avian embryos in ovo. Mol Imaging Biol. 2011;13: 208-214.
14. Würbach L, Heidrich A, Opfermann T, Gebhardt P, Saluz HP. Insights into bone metabolism of avian embryos in ovo via $3 \mathrm{D}$ and $4 \mathrm{D}{ }^{18} \mathrm{~F}$-fluoride positron emission tomography. Mol Imaging Biol. 2012;14:688-698.

15. Hassan SM, Siam AA, Mady ME, Cartwright AL. Incubation temperature for ostrich (Struthio camelus) eggs. Poult Sci. 2004;83:495-499.

16. van Schalkwyk SJ, Brand Z, Cloete SWP, Blood JR. The influence of different disinfection protocols on the hatching performance of ostrich eggs. In: Huchzermeyer FW, ed. Ratites in a Competitive World: Proceedings of the 2nd International Ratite Congress. Strand, South Africa: De Jongh's; 1998:158.

17. Deeming DC. Factors affecting hatchability during commercial incubation of ostrich (Struthio camelus) eggs. Br Poult Sci. 1995;36:51-65.

18. Deeming DC, Ar A. Factors affecting the success of commercial incubation. In: Deeming DC, ed. The Ostrich: Biology, Production and Health. Wallingford, U.K.: CAB International; 159-190.

19. Leary S, Underwood W, Antony R, et al. AVMA Guidelines for the Euthanasia of Animals. Schaumburg, IL: American Veterinary Medical Association; 2013:63.

20. Lee HJ, Kim YM, Ono T, Han JY. Genome modification technologies and their applications in avian species. Int J Mol Sci. 2017;18:E2245.

21. Gaitanis A, Kastis GA, Vlastou E, Bouziotis P, Verginis P, Anagnostopoulos CD. Investigation of image reconstruction parameters of the Mediso nanoScan PC small-animal PET/CT scanner for two different positron emitters under NEMA NU 4-2008 standards. Mol Imaging Biol. 2017;19:550-559.

22. Maatjens CM, van Roovert-Reijrink IAM, Engel B, van der Pol CW, Kemp B, van den Brand $\mathrm{H}$. Temperature during the last week of incubation. I. Effects on hatching pattern and broiler chicken embryonic organ development. Poult Sci. 2016;95:956-965.

23. Goernig M, Liehr M, Tute C, et al. Magnetocardiography based spatiotemporal correlation analysis is superior to conventional ECG analysis for identifying myocardial injury. Ann Biomed Eng. 2009;37:107-111.

24. Waschkies C, Nicholls F, Buschmann J. Comparison of medetomidine, thiopental and ketamine/midazolam anesthesia in chick embryos for in ovo magnetic resonance imaging free of motion artifacts. Sci Rep. 2015;5:15536.

25. Meganck JA, Liu B. Dosimetry in micro-computed tomography: a review of the measurement methods, impacts, and characterization of the Quantum GX imaging system. Mol Imaging Biol. 2017;19:499-511. 